OPEN ACCESS

Edited by:

Yang Yang,

Shanghai Jiao Tong University, China

Reviewed by:

Mohit Kumar Jolly,

Indian Institute of Science (IISC), India

Vishal Acharya,

Institute of Himalayan Bioresource

Technology (CSIR), India

*Correspondence:

$Y-h$. Taguch

tag@granular.com

Specialty section:

This article was submitted to

Computational Genomics,

a section of the journal

Frontiers in Genetics

Received: 20 March 2020

Accepted: 05 June 2020

Published: 20 August 2020

Citation:

Taguchi Yh and Turki T (2020) Universal Nature of Drug Treatment Responses in Drug-Tissue-Wide Model-Animal Experiments Using

Tensor Decomposition-Based Unsupervised Feature Extraction. Front. Genet. 11:695

doi: 10.3389/fgene.2020.00695

\section{Universal Nature of Drug Treatment Responses in Drug-Tissue-Wide Model-Animal Experiments Using Tensor Decomposition-Based Unsupervised Feature Extraction}

\author{
Y-h. Taguchi ${ }^{1 *}$ and Turki Turki ${ }^{2}$ \\ ${ }^{1}$ Department of Physics, Chuo University, Tokyo, Japan, ${ }^{2}$ Department of Computer Science, King Abdulaziz University, \\ Jeddah, Saudi Arabia
}

Gene expression profiles of tissues treated with drugs have recently been used to infer clinical outcomes. Although this method is often successful from the application point of view, gene expression altered by drugs is rarely analyzed in detail, because of the extremely large number of genes involved. Here, we applied tensor decomposition (TD)-based unsupervised feature extraction (FE) to the gene expression profiles of 24 mouse tissues treated with 15 drugs. TD-based unsupervised FE enabled identification of the common effects of 15 drugs including an interesting universal feature: these drugs affect genes in a gene-group-wide manner and were dependent on three tissue types (neuronal, muscular, and gastroenterological). For each tissue group, TD-based unsupervised FE enabled identification of a few tens to a few hundreds of genes affected by the drug treatment. These genes are distinctly expressed between drug treatments and controls as well as between tissues in individual tissue groups and other tissues. We also validated the assignment of genes to individual tissue groups using multiple enrichment analyses. We conclude that TD-based unsupervised FE is a promising method for integrated analysis of gene expression profiles from multiple tissues treated with multiple drugs in a completely unsupervised manner.

Keywords: tensor decomposition, unsupervised learning, gene expression profiles, gene selection, drug treatment

\section{BACKGROUND}

Drug design is a time-consuming and expensive process. Multiple coordinated experimental efforts, involving large-scale trial-and-error methods, are required to investigate new compounds. In general, this is due to the inherent difficulties in identifying novel therapeutic targets such as genes that cause disease. Even where potential target genes are identified robustly, it is difficult to find drug candidate compounds that successfully bind to the proteins they encode.

Computer-based methods have been introduced in an attempt to shorten the period of drug development and to reduce the expenses involved. The two major computer-aided drug design strategies are ligand-based drug design (LBDD) and structure-based drug design (SBDD). LBDD has various advantages including less required computational resources and better success rates for drug design. However, it also has the disadvantage of limited ability to find drug candidate 
compounds with low structural similarity to known drugs. To compensate for the weaknesses of LBDD, SBDD shows a greater ability to find drug candidate compounds lacking in structural similarity with known drugs. This is because SBDD tries to screen drug candidate compounds by investigating whether these can bind to target proteins. The weak point of SBDD is that it requires massive computational resources, and this prevents its application to large-scale screening, in which candidate drug compounds often number several million.

Considering the relatively low cost of obtaining gene expression profiles, a third computer-aided strategy has been proposed: gene expression profile-based drug design. In this strategy, the gene expression profiles of tissues/cell lines treated with candidate drug compounds are collected. The collected profiles are then compared with those of tissues/cell lines treated with known drug compounds. If the candidate drug compounds share a gene expression profile to some extent with known drug compounds, they are identified as having therapeutic potential against target diseases/proteins.

Some databases have been established to assist gene expression profiling for drug design. For example, chemical checker (DuranFrigola et al., 2020) includes gene expression in computer-aided drug design, whereas PharmacoDB (Smirnov et al., 2017) is fully implemented to consider the dose dependence of drugtreated cell lines for drug design. Many papers have been published on the use of gene expression profiles for computeraided drug design (Chengalvala et al., 2007; Bates, 2011). For instance, Huang et al. (2019) used combinatorial analysis of drug-induced gene expression for cancer drugs, which were then experimentally confirmed in vitro. Lee et al. (2017) proposed DeSigN, a robust and useful method for identifying candidate drugs using an input gene signature obtained from gene expression analysis. Kim et al. (2019) performed computational drug repositioning for gastric cancer using reversal of gene expression profiles, and De Wolf et al. (2018) analyzed highthroughput gene expression profiles to identify similarities between drugs and to predict compound activity. Hodos et al. (2017) tried to fill in missing gene expression observations in cells treated with drugs by predicting cell-specific drug perturbation profiles using available expression data from related conditions. Pabon et al. (2018) predicted protein targets for drug-like compounds using transcriptomics. In contrast, Liu et al. (2017) performed comparative analysis of genes that are frequently regulated by drugs based on connectivity to map transcriptome data.

In contrast to these successful applications of gene expression profile analysis to computer-aided drug design, it is unclear how individual gene expression is affected by drug treatment. First, the number of genes expressed in a dose dependent-manner is as large as the number of genes expressed. Thus, it is not easy to invent a useful method to integrate and understand the dose dependent-genes pertaining to individual gene expression profiles. For example, Lukačišin and Bollenbach (2019) employed principal component analysis (PCA) to integrate the dose dependence of gene expression profiles upon combinatorial drug treatment. They reported a convex (not monotonic) dependence on dose density and identified this as evidence of the cooperative effects of dual drug treatments. Nevertheless, convex dependence on dose was reportedly observed in a single drug treatment if tensor decomposition (TD) was employed to integrate multiple gene expression profiles of cell lines treated with a single drug (Taguchi, 2019). Thus, it is primarily important to identify an effective method that can integrate numerous gene expression profiles of tissues/cell lines treated with drugs.

Recently, Kozawa et al. (2020) used the gene expression profiles of mouse tissues treated with drugs to predict human clinical outcomes. In this paper, we applied TD-based unsupervised feature extraction (FE) to the gene expression profiles used in their study and attempted to identify the changes in gene expression profiles of mouse tissues treated with individual drugs.

\section{METHODS AND MATERIALS}

Figure 1 shows the flow chart of analysis.

\section{Gene Expression Profiles}

The gene expression profiles used in this study were downloaded from the gene expression omnibus (GEO) with GEO ID GSE142068. Twenty four profiles named "GSE142068_count_XXXXX.txt.gz" were downloaded, where "XXXXX" indicates one of the 24 tissues, i.e., AdrenalG, Aorta, BM (Bone marrow), Brain, Colon, Eye, Heart, Ileum, Jejunum, Kidney, Liver, Lung, Pancreas, ParotidG, PituitaryG, SkMuscle, Skin, Skull, Spleen, Stomach, Spleen, Thymus, ThyroidG, and WAT (white adipose tissue), which were treated with 15 drugs: Alendronate, Acetaminophen, Aripiprazole, Asenapine, Cisplatin, Clozapine, Clozapine, Empagliflozin, Lenalidomide, Lurasidone, Olanzapine, Evolocumab, Risedronate, Sofosbuvir, and Teriparatide, and Wild type (WT).

\section{TD-Based Unsupervised FE}

For applying TD-based unsupervised FE (Taguchi, 2020) to the downloaded gene expression profiles, they must be formatted as a tensor. In this analysis, they were formatted as tensor, $x_{i j k m} \in$ $\mathbb{R}^{N \times 24 \times 18 \times 2}$, for $N$ genes, 24 tissues, 18 drug treatments, and two replicates. Then, the HOSVD (Taguchi, 2020) algorithm was applied to $x_{i j k m}$ and we obtained TD

$$
x_{i j k m}=\sum_{\ell_{1} \ell_{2} \ell_{3} \ell_{4}} G\left(\ell_{1}, \ell_{2}, \ell_{3}, \ell_{4}\right) u_{\ell_{1} j} u_{\ell_{2} k} u_{\ell_{3} m} u_{\ell_{4} i}
$$

where $G \in \mathbb{R}^{N \times 24 \times 18 \times 2}$ is the core tensor, $u_{\ell_{1} j} \in \mathbb{R}^{24 \times 24}, u_{\ell_{2} k} \in$ $\mathbb{R}^{18 \times 18}, u_{\ell_{3} m} \in \mathbb{R}^{2 \times 2}$, and $u_{\ell_{4} i} \in \mathbb{R}^{N \times N}$, represents singular value matrices that are also orthogonal matrices. $x_{i j k m}$ is considered to be standardized as $\sum_{i} x_{i j k m}=0$ and $\sum_{i} x_{i j k m}^{2}=N$.

Mathematically, Equation (1) aims to decompose the dependence of $x_{i j k m}$ upon $i, j, k, m$ into a series of products among $u_{\ell_{1} j}, u_{\ell_{2} k}, u_{\ell_{3} m}$, and $u_{\ell_{4}}$, each of which is supposed to represent the dependence on $i, j, k, m$. As it is unlikely that a single product of $u_{\ell_{1} j}, u_{\ell_{2} k}, u_{\ell_{3} m}$, and $u_{\ell_{4} i}$ can reproduce $x_{i j k m}$, we need to consider various combinations of $u_{\ell_{1}}, u_{\ell_{2} k}, u_{\ell_{3} m}$, and $u_{\ell_{4} i}$, where those associated with distinct $\ell_{1}, \ell_{2}, \ell_{3}, \ell_{4}$ are supposed to be associated with distinct dependence on $i, j, k, m$. 


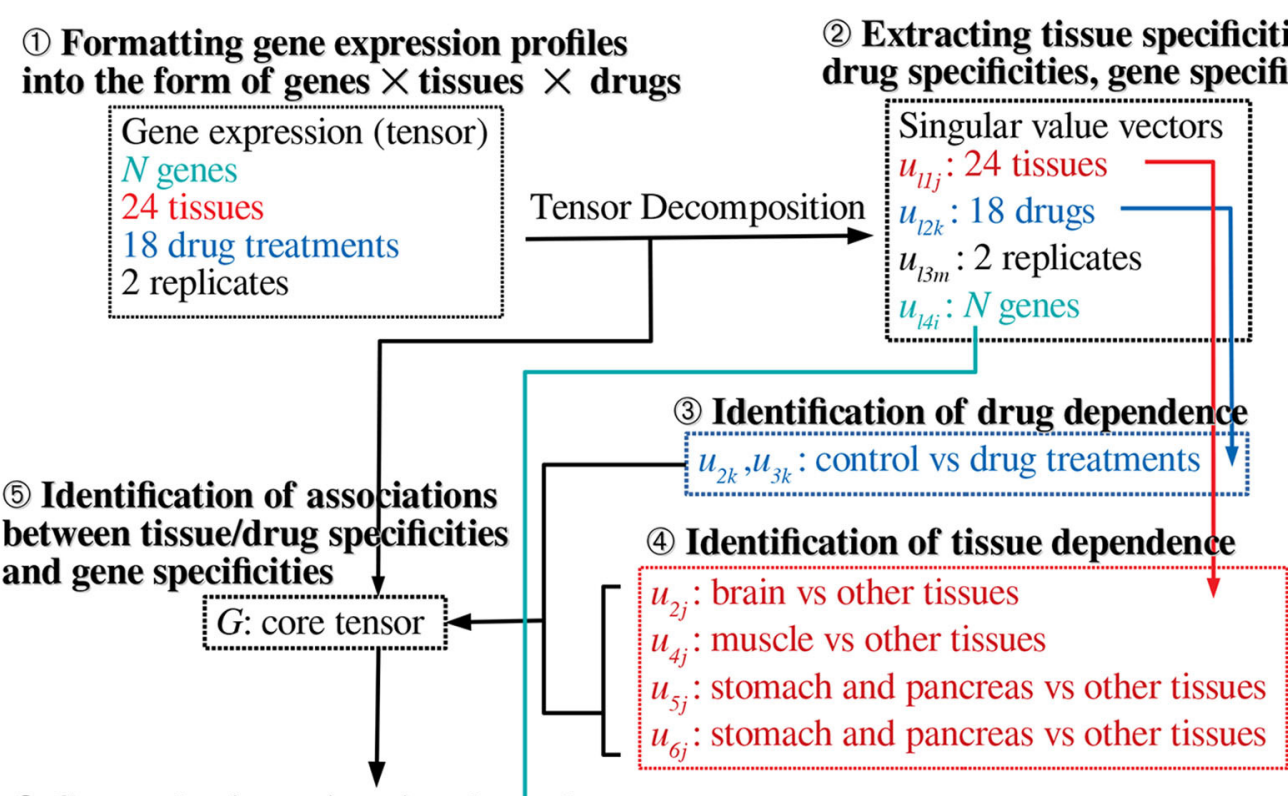

(6) Gene selection using singular value vectors identified to be associated with tissue/drug specificity

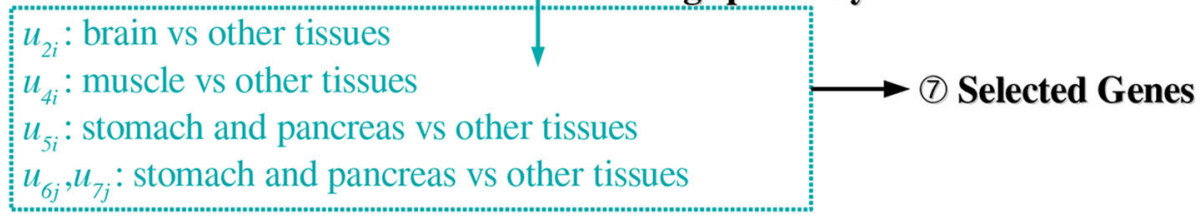

FIGURE 1 | Schematic of the analysis performed in this study.

Then, the products of $u_{\ell_{1} j}, u_{\ell_{2} k}, u_{\ell_{3} m}$, and $u_{\ell_{4} i}$, must be summed up with the weight of $G$ to reproduce $x_{i j k m}$. Biologically, we cannot expect that $u_{\ell_{1} j}, u_{\ell_{2} k}, u_{\ell_{3} m}$, and $u_{\ell_{4} i}$ can represent the biological aspect because Equation (1) is simply a mathematical hypothesis; therefore, their association with a biological aspect after performing TD needs to be validated.

To understand how gene expression profiles are altered by drug treatment in a tissue-group-wide manner, we first need to investigate $u_{\ell_{1} j}, u_{\ell_{2} k}$, and $u_{\ell_{3} m}$. After identifying which $\ell_{1}, \ell_{2}$, and $\ell_{3}$ are biologically interesting, we select $\ell_{4}$ associated with $G\left(\ell_{1}, \ell_{2}, \ell_{3}, \ell_{4}\right)$ that have the largest absolute values with fixed $\ell_{1}$, $\ell_{2}$, and $\ell_{3}$, because $u_{\ell_{4} i}$ associated with such $\ell_{4}$ is supposed to represent the weight of gene $i$ that is expressed in association with $j, k, m$ dependence represented by the selected $u_{\ell_{1}}, u_{\ell_{2} k}, u_{\ell_{3} m}$.

Using the identified $u_{\ell_{4}}$, the $P$-value, $P_{i}$, is attributed to gene $i$ as

$$
P_{i}=P_{\chi^{2}}\left[>\left(\frac{u_{\ell_{4} i}}{\sigma_{\ell_{4}}}\right)^{2}\right]
$$

where $P_{\chi^{2}}[>x]$ is the cumulative probability of $\chi^{2}$ distribution and $\sigma_{\ell_{4}}$ is the standard deviation. Here, we assume that $u_{\ell_{4} i}$ obeys a Gaussian distribution with zero mean because $\sum_{i} x_{i j k m}=$ 0. $P_{i}$ is corrected via the $\mathrm{BH}$ criterion (Burgos et al., 2014) and $I$, a set of genes $i$ associated with adjusted $P$-values less than 0.01, is selected. For a more detailed explanation of TD-based unsupervised FE, see the recently published monograph (Taguchi, 2020).

\section{$t$-Test and Wilcoxon Test Applied to Sets of Genes Classified Based on Tissue Groups and Drugs Groups}

In order to determine whether the selected set of genes, $I$, are expressed distinctly between the two assigned tissue groups, $J$, $\left\{x_{i j k m} \mid i \in I, j \in J\right\}$, and $\bar{J},\left\{x_{i j k m} \mid i \in I, j \in \bar{J}\right\}$, we applied a twoway $t$ test and Wilcoxon test and computed the $P$-values. Similar analyses were done with two drug groups, $K,\left\{x_{i j k m} \mid i \in I, k \in K\right\}$, and $\bar{K},\left\{x_{i j k m} \mid i \in I, k \in \bar{K}\right\}$.

\section{Enrichment Analysis}

The selected genes (gene symbols) were uploaded to Enrichr (Kuleshov et al., 2016) and Metascape (Zhou et al., 2019) in order to validate the various biological functions of the selected genes.

\section{RESULTS}

Figure 2 summarizes the results obtained in this study. 


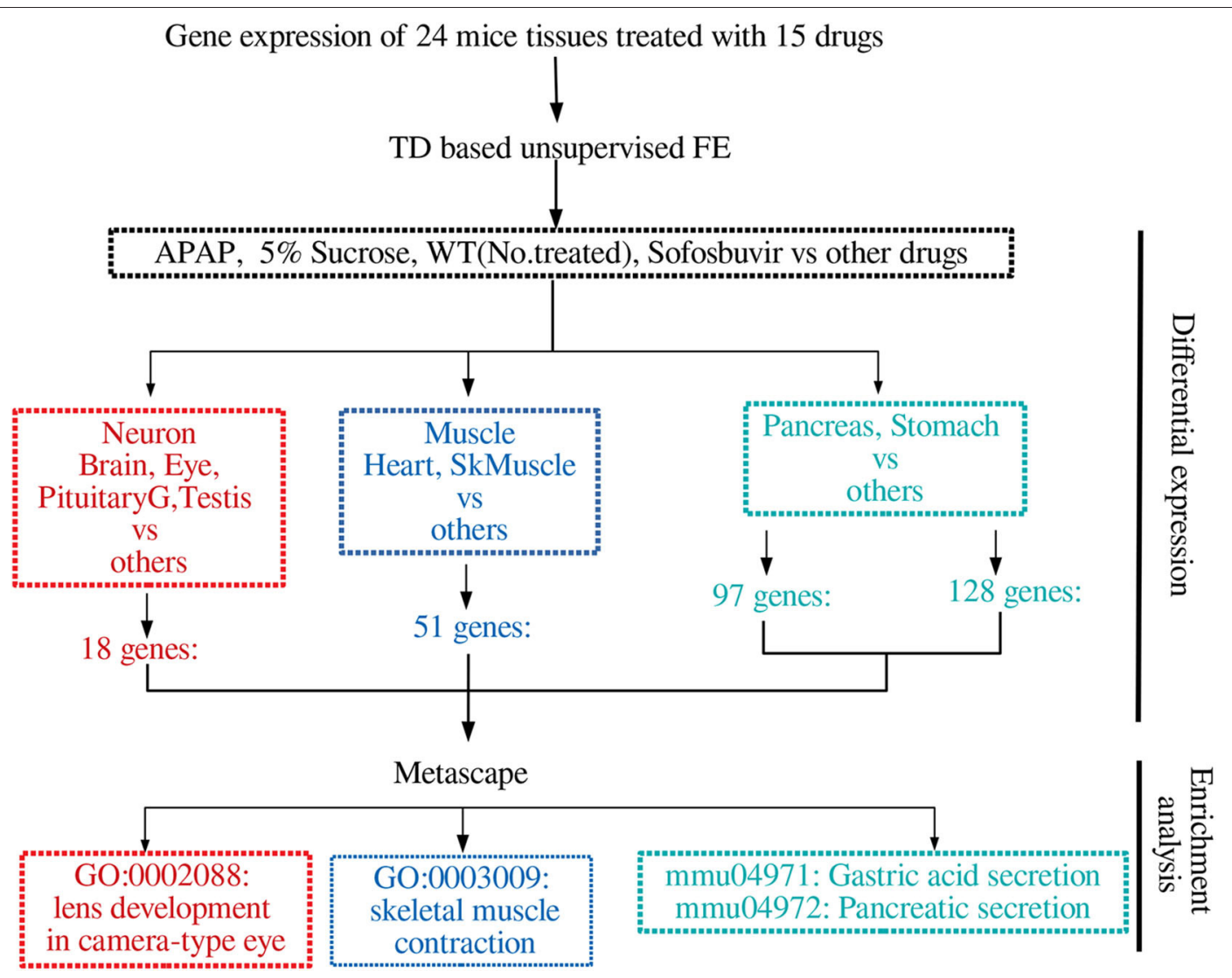

FIGURE 2 | Summary of the results obtained in this study.

\section{Drug Treatment Specificity}

After obtaining the TD, Equation (1), we first investigated $u_{\ell_{2} k}$ attributed to the $k$ th drug. Although the number of drugs tested is as many as 15 , the total number of drug treatments was considered to be 18 due to the testing of three additional conditions. Usually, the first singular value vectors represent uniform values (i.e., components that are not distinct between samples) (Taguchi, 2020). In this case, $u_{1 k}$ does not represent any dependence on drug treatment. This is reasonable because the expression of most genes is unlikely to be affected by drug treatment. We thus considered the second and third singular value vectors, $u_{2 k}$ and $u_{3 k}$, attributed to drug treatments (Figure 3). In contrast to expectations, the drug treatments were quite universal. Most of the drug treatments [other than (2), (9), (15), and (17)] were separated from the control treatments [(2), (9), (15), and (17)] along one direction (red arrow) whereas the diversity among drug treatments was spread perpendicular (blue arrow) to that direction, only among drug treatments. This suggests that the gene expression profiles are altered similarly, independently of the drug treatment.

\section{Tissue-Specificity}

We further studied the relationship of universal drug treatments with individual tissues. For this, we next investigated $u_{\ell_{1} j}$ attributed to 24 tissues. We then found that several $u_{\ell_{1} j}$ are expressed in a tissue-group wide manner (Figure 4). The tissuewide expression pattern identified by singular value vectors is described as follows; As $u_{1 j}$ does not express any tissue specificities, it is unlikely to exhibit tissue specificity; as $u_{2 j}$ has larger absolute values for the brain, eye, pituitary, and testis, it is likely to represent neuronal tissue specificities; as $u_{3 j}$ has larger absolute values only for the parotid, we did not consider it further; as $u_{4 j}$ exhibits larger absolute values for the heart and SkMuscle, we considered that it exhibits muscle specificities; As $u_{5 j}$ and $u_{6 j}$ exhibit larger absolute values for the pancreas and stomach, we considered that it exhibits gastric tissue specificities. It is thus obvious that the combination of tissue specificity is quite reasonable biologically.

Aiming to specify singular value vectors attributed to genes, $u_{\ell_{4}}$, for gene selection, we then checked which of $G\left(\ell_{1}, 2,1, \ell_{4}\right)$ and $G\left(\ell_{1}, 3,1, \ell_{4}\right)$ have larger absolute values, as $u_{1 m}$ always exhibits the same values between two replicates (Table 1).

For $\ell_{1}=2$, which is supposed to be attributed to neuronspecific tissues $\left(u_{2 j}\right)$, Gs with $\ell_{4}=2$ have larger absolute values. Thus, $u_{2 i}$ was employed for neuron-specific gene selection. For $\ell_{1}=4$, which is supposed to be attributed to muscle-specific tissues $\left(u_{4 j}\right)$, Gs with $\ell_{4}=4$ have larger absolute values. Thus, $u_{4 i}$ was employed for muscle-specific gene selection. For $\ell_{1}=5$, which is supposed to be attributed to gastrointestinal-specific tissues $\left(u_{5 j}\right)$, Gs with $\ell_{4}=5$ have larger absolute values. Thus, 


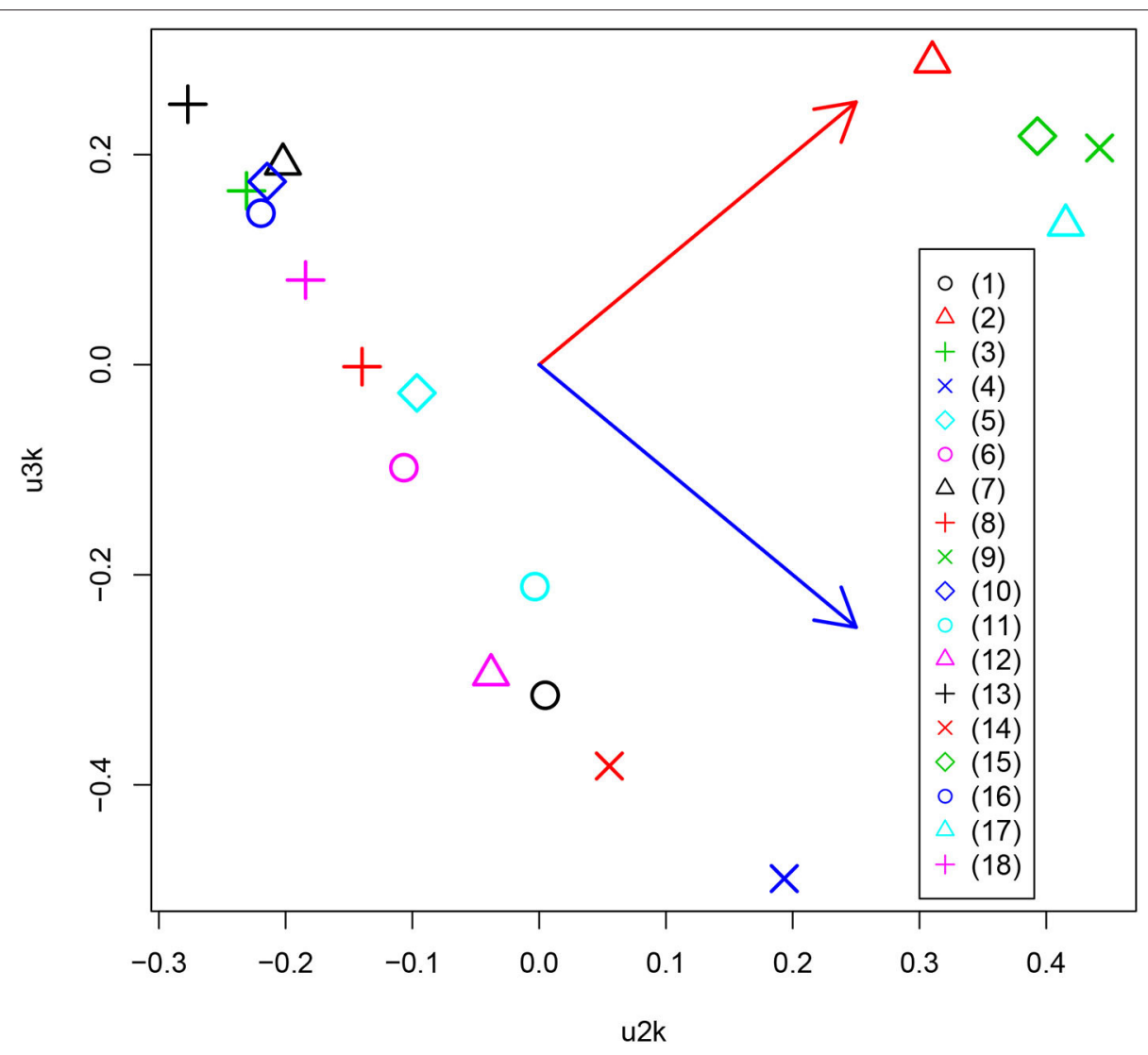

FIGURE 3 | Scatter plot between $u_{2 k}$ and $u_{3 k}$ attributed to drug treatment. Red and blue arrows represent distinct controls and drug treatments, and diversity among drug treatments, respectively. (1) Alendronate, (2) APAP, (3) Aripiprazole, (4) Asenapine, (5) Cisplatin, (6) Clozapine, (7) Dox, (8) EMPA, (9) FivePercentSucrose, (10) Lenalidomide, (11) Lurasidone, (12) Olanzapine, (13) Repatha, (14) Risedronate, (15) Sofosbuvir, (16) Teriparatide, (17) WT.No.treated, (18) 5 percentCMC0.25percentTween80.

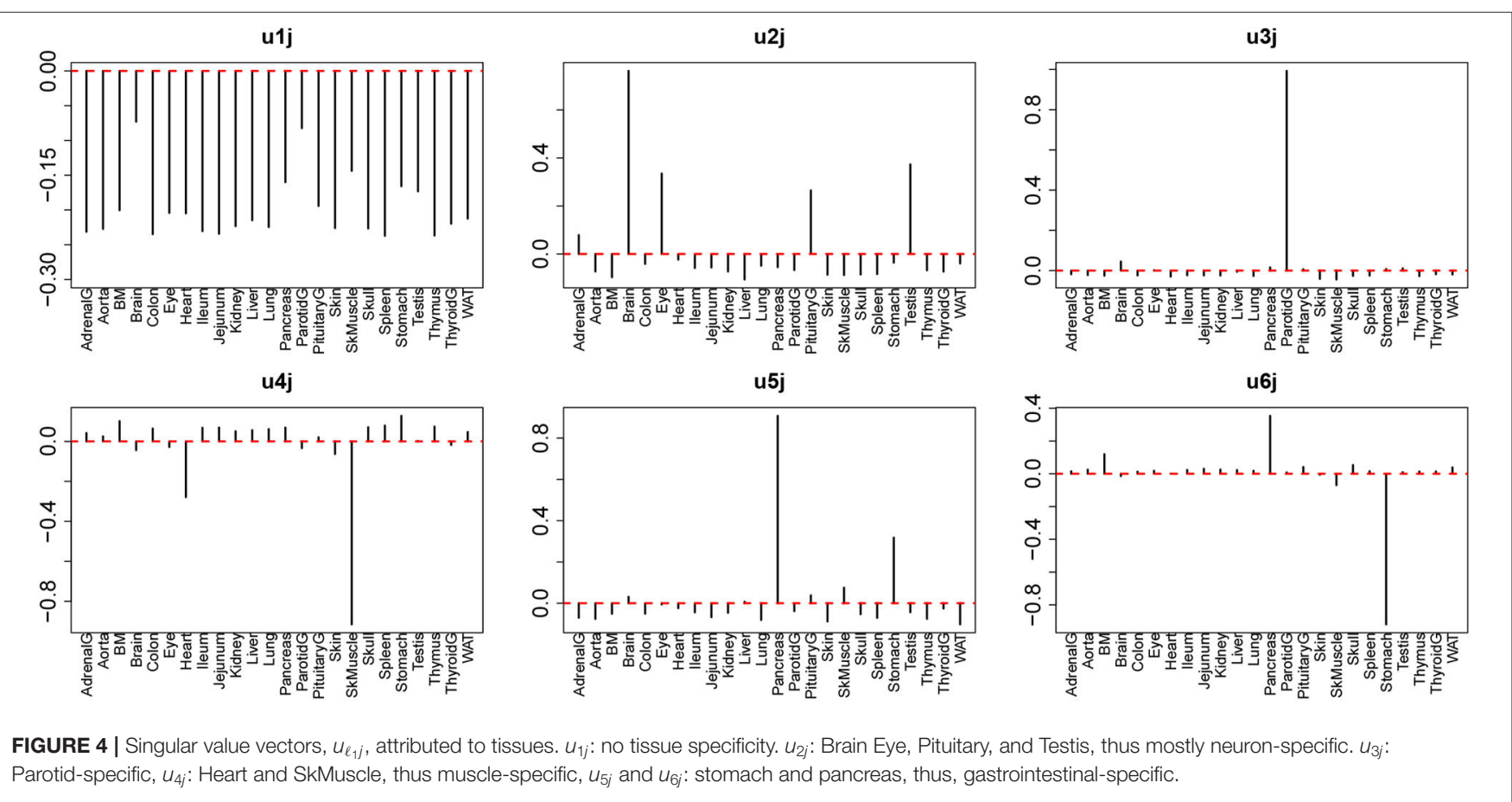


TABLE 1 | $G\left(\ell_{1}, 2,1, \ell_{4}\right)$ and $G\left(\ell_{1}, 3,1, \ell_{4}\right)$ for $\ell_{1}=2,4,5,6$.

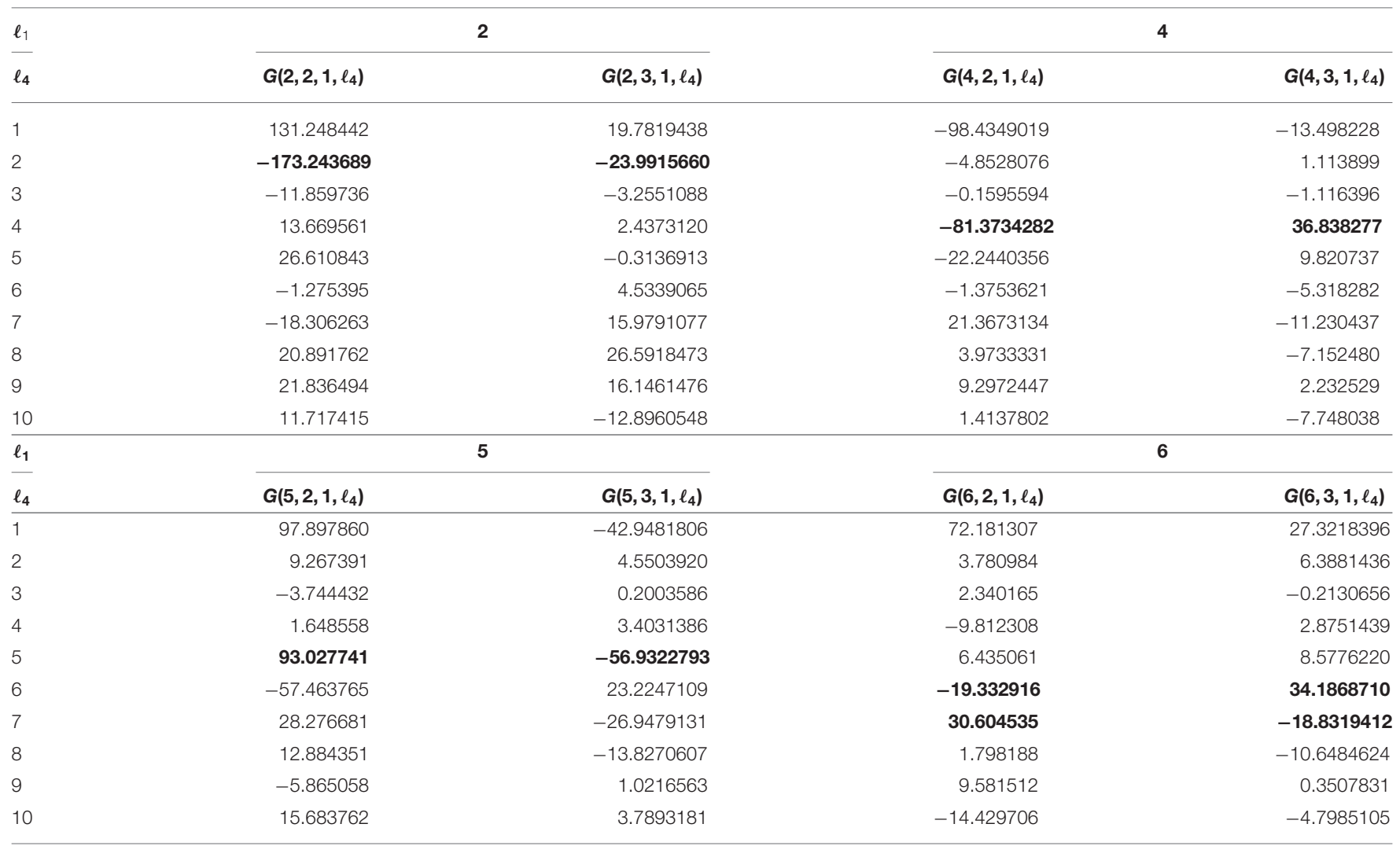

Values in bold correspond to those of $\ell_{4} s$ used for gene selection with $u_{\ell_{4}}$.

TABLE 2 | Statistical tests for distinct expression between the specified tissues and other tissues, and between drug treatments and controls.

\begin{tabular}{|c|c|c|c|c|c|c|}
\hline \multirow[b]{3}{*}{$\ell_{1}$} & \multirow[b]{3}{*}{$\begin{array}{l}\text { Tissue } \\
\text { specificity }\end{array}$} & \multirow[b]{3}{*}{$\begin{array}{l}\text { \# of } \\
\text { Genes }\end{array}$} & \multirow[b]{3}{*}{$\begin{array}{c}\text { Specified } \\
\text { tissues }\end{array}$} & \multicolumn{3}{|c|}{$\boldsymbol{P}$-values by statistical tests } \\
\hline & & & & \multirow{2}{*}{$\begin{array}{c}\text { Tissues } \\
t \text {-test }\end{array}$} & \multicolumn{2}{|c|}{ Drug treatment } \\
\hline & & & & & $\begin{array}{c}\text { Wilcoxon } \\
\text { test }\end{array}$ & $t$-test \\
\hline 2 & Neuron & 18 & $\begin{array}{l}\text { Brain, Eye, } \\
\text { Pituitary, } \\
\text { Testis }\end{array}$ & $2.14 \times 10^{-24}$ & $9.65 \times 10^{-49}$ & 0.22 \\
\hline 4 & Muscle & 51 & $\begin{array}{l}\text { Heart, } \\
\text { SkMuscle }\end{array}$ & $1.99 \times 10^{-55}$ & $2.67 \times 10^{-77}$ & 0.04 \\
\hline 5 & Gastrointestinal & 97 & $\begin{array}{l}\text { Pancreas, } \\
\text { Stomach }\end{array}$ & $8.48 \times 10^{-11}$ & $2.73 \times 10^{-40}$ & $8.13 \times 10^{-22}$ \\
\hline 6 & & 128 & & $6.67 \times 10^{-8}$ & $8.69 \times 10^{-90}$ & $8.69 \times 10^{-90}$ \\
\hline
\end{tabular}

$u_{5 i}$ was employed for muscle-specific gene selection. For $\ell_{1}=$ 6 , which is also supposed to be attributed to gastrointestinalspecific tissues $\left(u_{6 j}\right)$, Gs with $\ell_{4}=6,7$ have larger absolute values. Then, $u_{6 i}$ and $u_{7 i}$ were employed for muscle-specific gene selection.

After computing the adjusted $P$-values, $P_{i}$, attributed to the genes (see methods), the genes associated with adjusted $P_{i}$ $<0.01$ were selected (Table 2). The lists of selected genes can be found in supporting information (Additional File 1). Figure 5 shows a Venn diagram of the selected genes. As expected, two sets of genes, Gas1 and Gas2, which are supposed to be gastrointestinal-specific, are quite common. Other than these, the selected genes are quite distinct from one another. Thus, TD-based unsupervised FE successfully identified the genes whose expression was affected by the drugs in a tissue groupspecific manner. 


\section{Confirmation of Differential Expression}

In order to check whether the selected genes are expressed distinctly between the specified tissues and other tissues, as well as between drug treatments and controls, we first applied statistical

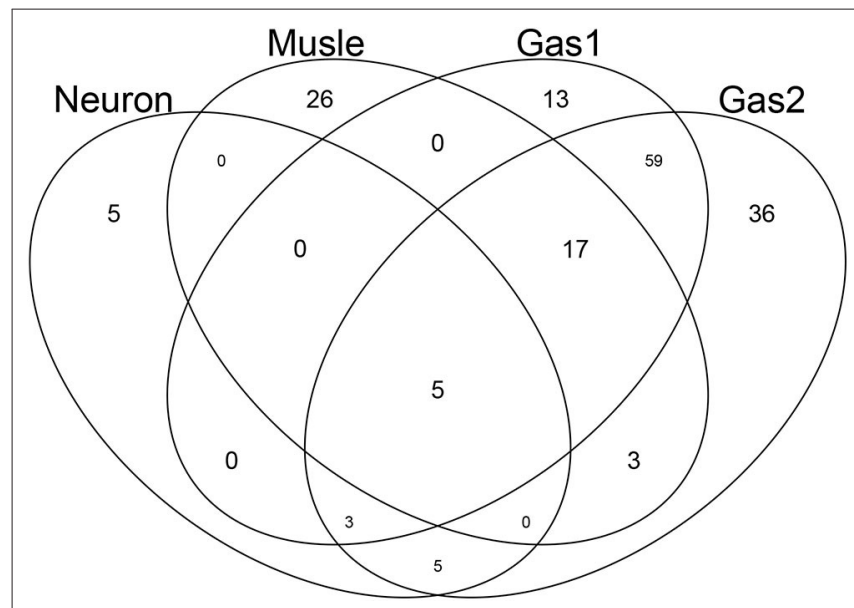

FIGURE 5 | Venn diagram of genes selected by TD-based unsupervised FE. Neuron: genes associated with $u_{2 j}$, which is supposed to be neuron-specific. Muscle: genes associated with $u_{4 j}$, which is supposed to be muscle-specific. Gas1 and Gas2: genes associated with $u_{5 j}$ and $u_{6 j}$, respectively, which are supposed to be gastrointestinal-specific. tests to the selected genes (Table 2). The data clearly showed that for all cases, gene expression was distinct between the specified tissues and other tissues as well as between drug treatments and

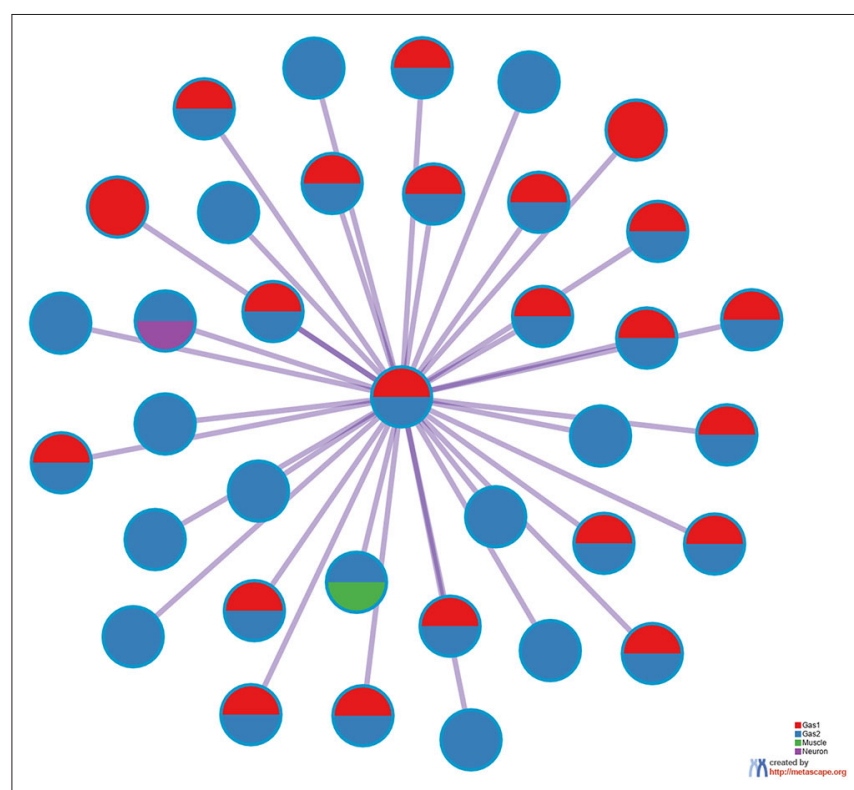

FIGURE 7 | PPI network provided by Metascape. Red: Gas1, Blue: Gas2, Green: Muscle, Purple: Neuron.

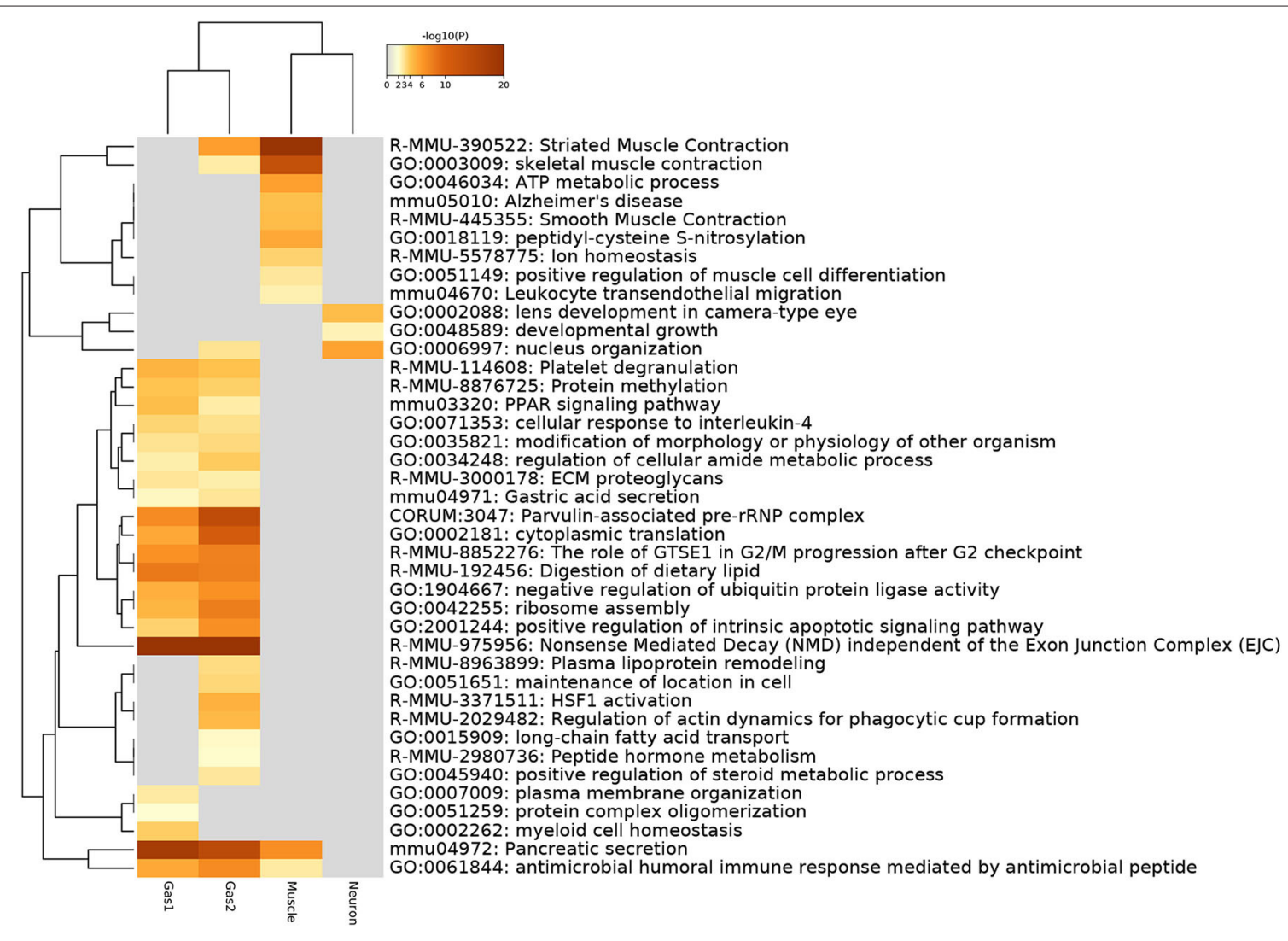

FIGURE 6 | Heatmap of enrichment analysis provided by Metascape. 
TABLE 3 | Enrichment analysis for "Disease Perturbations from GEO up" and "Disease Perturbations from GEO down" by Enrichr.

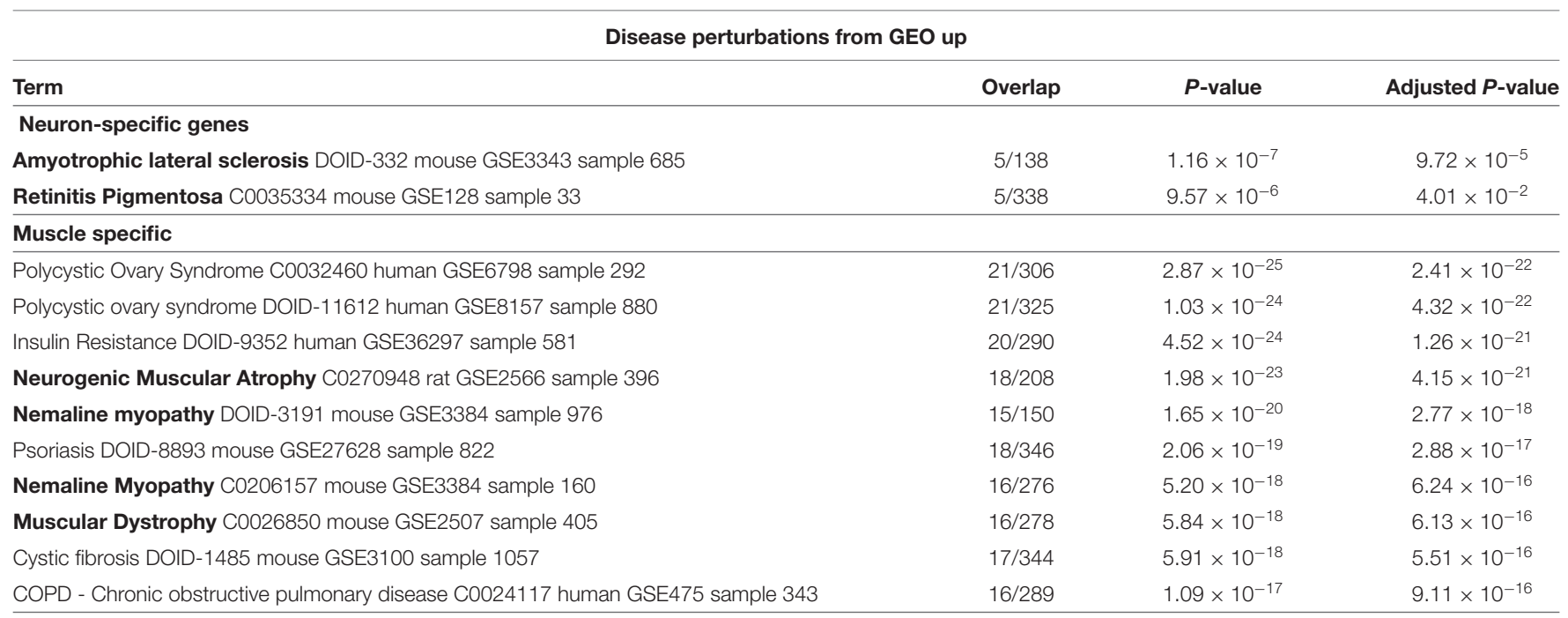

COPD - Chronic obstructive pulmonary disease C0024117 human GSE475 sample 343
Disease perturbations from GEO down

\begin{tabular}{|c|c|c|c|}
\hline Term & Overlap & $P$-value & Adjusted $P$-value \\
\hline \multicolumn{4}{|l|}{ Gas1 genes } \\
\hline Pancreatitis DOID-4989 mouse GSE3644 sample 513 & $36 / 238$ & $9.21 \times 10^{-45}$ & $7.73 \times 10^{-42}$ \\
\hline Pancreatic ductal adenocarcinoma DOID-3498 mouse GSE53659 sample 699 & 26/101 & $1.24 \times 10^{-38}$ & $3.48 \times 10^{-36}$ \\
\hline $\begin{array}{l}\text { Pancreatic invasive intraductal papillary-mucinous carcinoma DOID-8150 human } \\
\text { GSE19650 sample } 610\end{array}$ & $31 / 248$ & $1.21 \times 10^{-35}$ & $2.54 \times 10^{-33}$ \\
\hline Acute pancreatitis C0001339 mouse GSE3644 sample 376 & 28/188 & $2.33 \times 10^{-34}$ & $3.26 \times 10^{-32}$ \\
\hline Cystic Fibrosis C0010674 mouse GSE769 sample 428 & $31 / 275$ & $3.34 \times 10^{-34}$ & $4.00 \times 10^{-32}$ \\
\hline Chronic phase chronic myelogenous leukemia DOID-8552 human GSE5550 sample 456 & $30 / 270$ & $7.05 \times 10^{-33}$ & $7.40 \times 10^{-31}$ \\
\hline Invasive ductal carcinoma DOID-3008 human GSE21422 sample 606 & $31 / 304$ & $8.09 \times 10^{-33}$ & $7.54 \times 10^{-31}$ \\
\hline Eczema C0013595 human GSE6012 sample 268 & $26 / 163$ & $1.06 \times 10^{-32}$ & $8.87 \times 10^{-31}$ \\
\hline Systemic lupus erythematosus (SLE) DOID-9074 human GSE36700 sample 512 & $47 / 294$ & $1.50 \times 10^{-53}$ & $3.15 \times 10^{-51}$ \\
\hline Invasive ductal carcinoma DOID-3008 human GSE21422 sample 606 & $44 / 304$ & $5.90 \times 10^{-48}$ & $9.90 \times 10^{-46}$ \\
\hline Eczema C0013595 human GSE6012 sample 268 & $37 / 163$ & $7.47 \times 10^{-48}$ & $1.04 \times 10^{-45}$ \\
\hline Malignant Melanoma C0025202 human GSE3189 sample 117 & $41 / 250$ & $6.75 \times 10^{-47}$ & $8.09 \times 10^{-45}$ \\
\hline Chronic phase chronic myelogenous leukemia DOID-8552 human GSE5550 sample 456 & $41 / 270$ & $1.91 \times 10^{-45}$ & $2.00 \times 10^{-43}$ \\
\hline Sickle Cell Anemia C0002895 human GSE9877 sample 109 & $37 / 197$ & $1.61 \times 10^{-44}$ & $1.50 \times 10^{-42}$ \\
\hline Actinic keratosis C0022602 human GSE2503 sample 350 & $46 / 429$ & $4.69 \times 10^{-44}$ & $3.94 \times 10^{-42}$ \\
\hline
\end{tabular}

Diseases in bold correspond to those related to specific tissues. Up to the top 10 ranked terms are shown.

controls. Thus, TD-based unsupervised FE allowed us to select the genes whose expression is coincident with $u_{\ell_{2} k}$ in Figure 3 and $u_{\ell_{1 j}}$ in Figure 4.

\section{Biological Evaluation}

Next, we evaluated the selected genes biologically. For this purpose, we first uploaded the genes to Metascape (Figure 6). Initially, we noticed that Gas1 and Gas2 largely shared the enriched terms as expected, even though these two gene sets were selected using distinct singular values $\left(u_{5 i}\right.$ and $u_{6 i}, u_{7 i}$, respectively). In particular, it is important to note that two KEGG terms, "mmu04971: Gastric acid secretion" and "mmu04972: Pancreatic secretion" are shared by Gas1 and Gas2, which are supposed to be Pancreas- and Stomach-specific. In contrast, various muscle-related terms are enriched in the Muscle gene set as expected, whereas "GO:0002088: lens development in camera-type eye" is enriched in the neuronal gene set. All of these results suggest 
TABLE 4 | Previously reported drug effects on neuron (brain and eye), muscle and pancreas tissues.

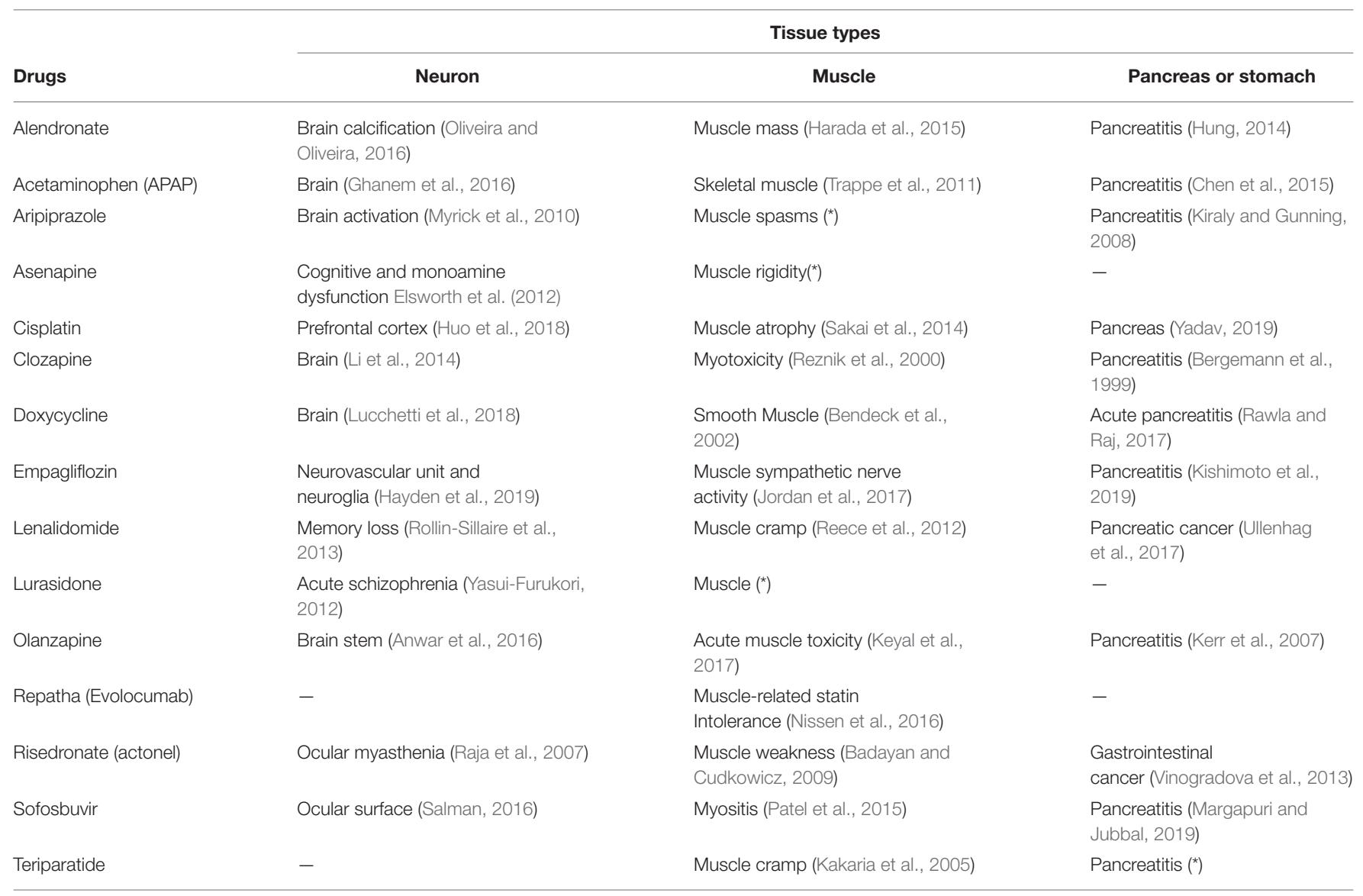

${ }^{*}$ Reported side effects.

that TD-based unsupervised FE selected the biologically reasonable genes.

Figure 7 shows the protein-protein interaction (PPI) network provided by Metascape. A high degree of connectivity was obvious. Thus, TD-based unsupervised FE identified the sets of genes among which PPI is enriched. Moreover, Gas1 and Gas2 largely share the PPI network, whereas the neuronal and muscular gene sets form their own PPI network within which PPI is enriched. Thus, PPI analysis also indicated that TD-based unsupervised FE identified biologically reasonable genes.

To eliminate the possibility that our results were specific to the Metascape data set, we uploaded the genes selected by TD-based unsupervised FE to Enrichr (Table 3). With this data set, we observed clear detection of at least one tissuerelated disease for four sets of tissue-specific genes, validating the Metascape-based results.

\section{DISCUSSION}

Although it is unclear why the 15 drugs affected the expression of many common genes, a detailed investigation can allow further interpretation. Table 4 shows the drugs' effects on neuronal, muscular, and pancreatic tissues. These data suggest that most drugs simultaneously affect these three groups of tissues.
Our results are in contrast to the study that inspired our work (Kozawa et al., 2020), in which the authors employed a fully supervised approach requiring previous knowledge. Although Kozawa et al. (2020) also aimed to infer the therapeutic and side effects of drug treatments in humans based on gene expression in drug-treated tissues, their analysis required previous knowledge that is not needed for TD-based unsupervised FE. In this sense, our approach has distinct potential that the original study could not achieve.

In addition to the above-mentioned biological superiority of TD-based unsupervised FE, this approach also has some methodological advantages as follows. First, although we classified 24 tissues into two groups based on the observation of singular value vectors attributed to tissues, $u_{\ell_{1} j}$ (Figure 4) prior to the identification of differentially expressed genes, it is computationally infeasible for other methods to classify 24 tissues into two groups before starting to seek differentially expressed genes, as there are no criteria on how to divide 24 tissues into two groups. It is thus practically impossible to analyze all possible divisions, as they number in the millions. The same advantage is observed when grouping 18 drug treatments into two. This may be much easier than classifying tissues, because some of the drug treatments are obviously controls. Nevertheless, based upon the second and 
third singular value vectors attributed to drug treatments, $u_{2 k}$ and $u_{3 k}$ (Figure 3), acetaminophen (APAP) and sofosbuvir are grouped together with two control treatments. Such a classification can never be proposed without TD. In this sense, there is no computationally feasible method that can compete with our method.

The biological basis for the two groups of drugs seen in Figure 3 may be questioned. To clarify this point, we uploaded two groups of drugs to DrugEnrichr (Kuleshov et al., 2020), which evaluates the coincidence of genes targeted by the uploaded drugs (Additional File 2). Based on the "Geneshot Predicted from Co-expression" category in DrugEnrichr, we found that there are at least as many as 164 genes targeted by two drugs (APAP and Sofosbuvir) in group1 whereas 213 genes are targeted by at least two drugs among as many as 13 drugs included in group2 (Alendronate, Aripiprazole, Asenapine, Cisplatin, Clozapine, Dox, EMPA, Lenalidomide, Lurasidone, Olanzapine, Repatha, Risedronate, Teriparatide). This suggests that these two groups of drugs are quite distinct because there are no common targeted genes between these 164 and 213 genes. Thus, the groups of drugs identified by TD based unsupervised FE are likely based on the genes that the drugs target.

In view of the two above-mentioned advantages, TDbased unsupervised FE might yield completely distinct outcomes that other supervised methods cannot, and it therefore represents a worthwhile primary or supplementary approach to gene-expression-based investigation of drug effects.

One might wonder if the results were confirmed only by single experiments. As the results shown in Table 3 indicate coincidence between the present result and other studies, the results derived in this study are not dependent on a single study, but are coincident with numerous studies in the public domain database.

Moreover, TD-based unsupervised FE is a very useful strategy for repositioning known drugs. As shown in Figure 3, TDbased unsupervised $\mathrm{FE}$ can determine the effective tissue. Furthermore, as indicated in Table 3, the genes selected by TDbased unsupervised FE can indicate the diseases for which the drugs have potential effectiveness. Therefore, applying TD-based unsupervised $\mathrm{FE}$ to gene expression profiles altered by drug treatments can be a promising strategy to repurpose known drugs for new diseases.

\section{REFERENCES}

Anwar, I. J., Miyata, K., and Zsombok, A. (2016). Brain stem as a target site for the metabolic side effects of olanzapine. J. Neurophysiol. 115, 1389-1398. doi: 10.1152/jn.00387.2015

Badayan, I., and Cudkowicz, M. E. (2009). Profound muscle weakness and pain after one dose of Actonel. Case Rep. Med. 2009:693014. doi: $10.1155 / 2009 / 693014$

Bates, S. (2011). The role of gene expression profiling in drug discovery. Curr. Opin. Pharmacol. 11, 549-556. doi: 10.1016/j.coph.2011.06.009

Bendeck, M. P., Conte, M., Zhang, M., Nili, N., Strauss, B. H., and Farwell, S. M. (2002). Doxycycline modulates smooth muscle cell growth, migration,

\section{CONCLUSIONS}

In this paper, we applied TD-based unsupervised FE (Taguchi, 2020) to the gene expression profiles of 24 mouse tissues treated with 15 drugs. Integrated analysis allowed us to identify the universal nature of drug treatments in a tissue-group-wide manner, which is generally impossible to identify using any other supervised strategy that requires prior information.

\section{DATA AVAILABILITY STATEMENT}

All datasets analyzed in this study were obtained from GEO: GSE142068.

\section{AUTHOR CONTRIBUTIONS}

Y-hT planned and performed the study. Y-hT and TT discussed the results and wrote the paper. All authors contributed to the article and approved the submitted version.

\section{FUNDING}

This study was supported by KAKENHI 19H05270, 20K12067, and $20 \mathrm{H} 04848$. This project was also funded by the Deanship of Scientific Research (DSR) at King Abdulaziz University, Jeddah, under grant no. KEP-8-611-38. The authors, therefore, acknowledge DSR with thanks for providing technical and financial support.

\section{ACKNOWLEDGMENTS}

The authors would like to thank the reviewers for very constructive comments and thoughtful suggestions. This manuscript has been released as a pre-print at BioRxiv (Taguchi and Turki, 2020).

\section{SUPPLEMENTARY MATERIAL}

The Supplementary Material for this article can be found online at: https://www.frontiersin.org/articles/10.3389/fgene. 2020.00695/full\#supplementary-material

Additional File 1 | List of genes selected by TD-based unsupervised FE. List of genes shown in Table 2 (xlsx).

Additional File 2 | List of genes predicted by DrugEnrichr, whose expression is likely affected by the drugs investigated in this study. List of genes (xlsx).

and matrix remodeling after arterial injury. Am. J. Pathol. 160, 1089-1095. doi: 10.1016/S0002-9440(10)64929-2

Bergemann, N., Ehrig, C., Diebold, K., Mundt, C., and Einsiedel, R. (1999). Asymptomatic pancreatitis associated with clozapine. Pharmacopsychiatry 32, 78-80. doi: 10.1055/s-2007-979197

Burgos, K., Malenica, I., Metpally, R., Courtright, A., Rakela, B., Beach, T., et al. (2014). Profiles of extracellular miRNA in cerebrospinal fluid and serum from patients with Alzheimer's and Parkinson's diseases correlate with disease status and features of pathology. PLoS ONE 9:e94839. doi: 10.1371/journal.pone.0094839

Chen, S.-J., Lin, C.-S., Hsu, C.-W., Lin, C.-L., and Kao, C.H. (2015). Acetaminophen poisoning and risk of acute 
pancreatitis. Medicine 94:e1195. doi: 10.1097/MD.00000000000 01195

Chengalvala, M. V., Chennathukuzhi, V. M., Johnston, D. S., Stevis, P. E., and Kopf, G. S. (2007). Gene expression profiling and its practice in drug development. Curr. Genomics 8, 262-270. doi: 10.2174/138920207781386942

De Wolf, H., Cougnaud, L., Van Hoorde, K., De Bondt, A., Wegner, J. K., Ceulemans, H., et al. (2018). High-throughput gene expression profiles to define drug similarity and predict compound activity. ASSAY Drug Dev. Technol. 16, 162-176. doi: 10.1089/adt.2018.845

Duran-Frigola, M., Pauls, E., Guitart-Pla, O., Bertoni, M., Alcalde, V., Amat, D., et al. (2020). Extending the small molecule similarity principle to all levels of biology with the Chemical Checker. Nat. Biotechnol. 34:591. doi: 10.1038/s41587-020-0564-6

Elsworth, J. D., Groman, S. M., Jentsch, J. D., Valles, R., Shahid, M., Wong, E., et al. (2012). Asenapine effects on cognitive and monoamine dysfunction elicited by subchronic phencyclidine administration. Neuropharmacology 62, 1442-1452. doi: 10.1016/j.neuropharm.2011.08.026

Ghanem, C. I., Pérez, M. J., Manautou, J., and Mottino, A. D. (2016). Acetaminophen from liver to brain: new insights into drug pharmacological action and toxicity. Pharmacol. Res. 109, 119-131. doi: 10.1016/j.phrs.2016.02.020

Harada, A., Ito, S., Matsui, Y., Sakai, Y., Takemura, M., Tokuda, H., et al. (2015). Effect of alendronate on muscle mass: Investigation in patients with osteoporosis. Osteopor. Sarcopen. 1, 53-58. doi: 10.1016/j.afos.2015.07.005

Hayden, M. R., Grant, D. G., Aroor, A. R., and DeMarco, V. G. (2019). Empagliflozin ameliorates type 2 diabetes-induced ultrastructural remodeling of the neurovascular unit and neuroglia in the female $\mathrm{db} / \mathrm{db}$ mouse. Brain Sci. 9:57. doi: 10.3390/brainsci9030057

Hodos, R., Zhang, P., Lee, H.-C., Duan, Q., Wang, Z., Clark, N. R., et al. (2017). "Cell-specific prediction and application of drug-induced gene expression profiles," in Biocomputing 2018, eds R. B. Altman, A. K. Dunker, L. Hunter, M.D. Ritchie, T. Murray, and T. E. Klein (Kohala Coast, HI: World Scientific), 32-43.

Huang, C.-T., Hsieh, C.-H., Chung, Y.-H., Oyang, Y.-J., Huang, H.-C., and Juan, H.-F. (2019). Perturbational gene-expression signatures for combinatorial drug discovery. iScience 15, 291-306. doi: 10.1016/j.isci.2019.04.039

Hung, W. Y. (2014). Contemporary review of drug-induced pancreatitis: A different perspective. World J. Gastrointest. Pathophysiol. 5:405. doi: 10.4291/wjgp.v5.i4.405

Huo, X., Reyes, T. M., Heijnen, C. J., and Kavelaars, A. (2018). Cisplatin treatment induces attention deficits and impairs synaptic integrity in the prefrontal cortex in mice. Sci. Rep. 8:17400. doi: 10.1038/s41598-018-35919-x

Jordan, J., Tank, J., Heusser, K., Heise, T., Wanner, C., Heer, M., et al. (2017). The effect of empagliflozin on muscle sympathetic nerve activity in patients with type II diabetes mellitus. J. Am. Soc. Hypertens. 11, 604-612. doi: 10.1016/j.jash.2017.07.005

Kakaria, P. J., Nashel, D. J., and Nylen, E. S. (2005). Debilitating muscle cramps after teriparatide therapy. Ann. Intern. Med. 142:310. doi: 10.7326/0003-4819-142-4-200502150-00023

Kerr, T. A., Jonnalagadda, S., Prakash, C., and Azar, R. (2007). Pancreatitis following olanzapine therapy: a report of three cases. Case Rep. Gastroenterol. 1, 15-20. doi: 10.1159/000104222

Keyal, N., Shrestha, G., Pradhan, S., Maharjan, R., Acharya, S., and Marhatta, M. (2017). Olanzapine overdose presenting with acute muscle toxicity. Int. J. Crit. Illness Inj. Sci. 7, 69-71. doi: 10.4103/2229-5151.201962

Kim, I.-W., Jang, H., Kim, J. H., Kim, M. G., Kim, S., and Oh, J. M. (2019). Computational drug repositioning for gastric cancer using reversal gene expression profiles. Sci. Rep. 9:2660. doi: 10.1038/s41598-019-39228-9

Kiraly, B., and Gunning, K. (2008). A case of pancreatitis associated with aripiprazole in the absence of hyperglycemia. Prim. Care Compan. J. Clin. Psychiatry 10, 484-485. doi: 10.4088/PCC.v10n0612e

Kishimoto, M., Yamaoki, K., and Adachi, M. (2019). Combination therapy with empagliflozin and insulin results in successful glycemic control: A case report of uncontrolled diabetes caused by autoimmune pancreatitis and subsequent steroid treatment. Case Rep. Endocrinol. 2019:9415347. doi: 10.1155/2019/9415347

Kozawa, S., Sagawa, F., Endo, S., Almeida, G. M. D., Mitsuishi, Y., and Sato, T. N. (2020). Predicting human clinical outcomes using mouse multi-organ transcriptome. iScience 23:100791. doi: 10.1016/j.isci.2019.100791
Kuleshov, M., Kropiwnicki, E., and Ma'ayan, A. (2020). Drugenrichr. Available online at: https://amp.pharm.mssm.edu/DrugEnrichr/

Kuleshov, M. V., Jones, M. R., Rouillard, A. D., Fernandez, N. F., Duan, Q., Wang, Z., et al. (2016). Enrichr: a comprehensive gene set enrichment analysis web server 2016 update. Nucleic Acids Res. 44, W90-W97. doi: 10.1093/nar/ gkw377

Lee, B. K. B., Tiong, K. H., Chang, J. K., Liew, C. S., Rahman, Z. A. A., Tan, A. C., et al. (2017). DeSigN: connecting gene expression with therapeutics for drug repurposing and development. BMC Genomics 18:934. doi: 10.1186/s12864-016-3260-7

Li, C. H., Stratford, R. E., de Mendizabal, N. V., Cremers, T. I., Pollock, B. G., Mulsant, B. H., et al. (2014). Prediction of brain clozapine and norclozapine concentrations in humans from a scaled pharmacokinetic model for rat brain and plasma pharmacokinetics. J. Transl. Med. 12:203. doi: 10.1186/1479-5876-12-203

Liu, X., Zeng, P., Cui, Q., and Zhou, Y. (2017). Comparative analysis of genes frequently regulated by drugs based on connectivity map transcriptome data. PLoS ONE 12:e179037. doi: 10.1371/journal.pone.0179037

Lucchetti, J., Fracasso, C., Balducci, C., Passoni, A., Forloni, G., Salmona, M., et al. (2018). Plasma and brain concentrations of doxycycline after single and repeated doses in wild-type and APP23 mice. J. Pharmacol. Exp. Therap. 368, 32-40. doi: 10.1124/jpet.118.252064

Lukačišin, M., and Bollenbach, T. (2019). Emergent gene expression responses to drug combinations predict higher-order drug interactions. Cell Syst. 9, 423-433.e3. doi: 10.1016/j.cels.2019.10.004

Margapuri, J., and Jubbal, S. (2019). 902: Acute pancreatitis following treatment with ledipasvir/sofosbuvir for hepatitis virus infection. Crit. Care Med. 47:430. doi: 10.1097/01.ccm.0000551651.45733.80

Myrick, H., Li, X., Randall, P. K., Henderson, S., Voronin, K., and Anton, R. F. (2010). The effect of aripiprazole on cue-induced brain activation and drinking parameters in alcoholics. J. Clin. Psychopharmacol. 30, 365-372. doi: 10.1097/JCP.0b013e3181e75cff

Nissen, S. E., Stroes, E., Dent-Acosta, R. E., Rosenson, R. S., Lehman, S. J., Sattar, N., et al. (2016). Efficacy and tolerability of evolocumab vs. ezetimibe in patients with muscle-related statin intolerance: the GAUSS-3 randomized clinical trial. JAMA 315, 1580-1590. doi: 10.1001/jama.2016.3608

Oliveira, J. R. M., and Oliveira, M. F. (2016). Primary brain calcification in patients undergoing treatment with the biphosphanate alendronate. Sci. Rep. 6:22961. doi: 10.1038/srep22961

Pabon, N. A., Xia, Y., Estabrooks, S. K., Ye, Z., Herbrand, A. K., Evelyn, S., et al. (2018). Predicting protein targets for drug-like compounds using transcriptomics. PLoS Comput. Biol. 14:e1006651. doi: 10.1371/journal.pcbi.1006651

Patel, S., Trakroo, S., Sanaka, S., and Qureshi, K. (2015). Severe myositis with the use of Sofosbuvir/Ledipasvir for Hepatitis C infection: a case of unexpected interactions. Am. J. Gastroenterol. 110:S333. doi: 10.14309/00000434-201510001-00761

Raja, V., Sandanshiv, P., and Neugebauer, M. (2007). Risedronate induced transient ocular myasthenia. J. Postgrad. Med. 53:274. doi: 10.4103/0022-3859.37525

Rawla, P., and Raj, J. P. (2017). Doxycycline-induced acute pancreatitis: a rare adverse event. Gastroenterol. Res. 10, 244-246. doi: 10.14740/gr838w

Reece, D., Kouroukis, C. T., LeBlanc, R., Sebag, M., Song, K., and Ashkenas, J. (2012). Practical approaches to the use of lenalidomide in multiple myeloma: a canadian consensus. Adv. Hematol. 2012:621958. doi: 10.1155/2012/ 621958

Reznik, I., Volchek, L., Mester, R., Kotler, M., Sarova-Pinhas, I., Spivak, B., et al. (2000). Myotoxicity and neurotoxicity during clozapine treatment. Clin. Neuropharmacol. 23, 276-280. doi: 10.1097/00002826-200009000-00007

Rollin-Sillaire, A., Delbeuck, X., Pollet, M., Mackowiak, M.-A., Lenfant, P., Noel, M.-P., et al. (2013). Memory loss during lenalidomide treatment: a report on two cases. BMC Pharmacol. Toxicol. 14:41. doi: 10.1186/2050-651114-41

Sakai, H., Sagara, A., Arakawa, K., Sugiyama, R., Hirosaki, A., Takase, K., et al. (2014). Mechanisms of cisplatin-induced muscle atrophy. Toxicol. Appl. Pharmacol. 278, 190-199. doi: 10.1016/j.taap.2014.05.001

Salman, A. G. (2016). Ocular surface changes with sofosbuvir in egyptian patients with Hepatitis C virus infection. Cornea 35, 323-328. doi: $10.1097 /$ ICO.0000000000000736 
Smirnov, P., Kofia, V., Maru, A., Freeman, M., Ho, C., El-Hachem, N., et al. (2017). PharmacoDB: an integrative database for mining in vitro anticancer drug screening studies. Nucleic Acids Res. 46, D994-D1002. doi: 10.1093/nar/ gkx911

Taguchi, Y-h. (2019). Drug candidate identification based on gene expression of treated cells using tensor decomposition-based unsupervised feature extraction for large-scale data. BMC Bioinformatics 19:388. doi: 10.1186/s12859-018-2395-8

Taguchi, Y-h. (2020). Unsupervised Feature Extraction Applied to Bioinformatics: A PCA Based and TD Based Approach. Switzerland: Springer International Publishing.

Taguchi, Y-h., and Turki, T. (2020). Universal nature of drug treatment responses in drug-tissue-wide model-animal experiments using tensor decompositionbased unsupervised feature extraction. bioRxiv 2020.03.08.982405. doi: 10.1101/2020.03.08.982405

Trappe, T. A., Carroll, C. C., Dickinson, J. M., LeMoine, J. K., Haus, J. M., Sullivan, B. E., et al. (2011). Influence of acetaminophen and ibuprofen on skeletal muscle adaptations to resistance exercise in older adults. Am. J. Physiol. Regul. Integr. Compar. Physiol. 300, R655-R662. doi: 10.1152/ajpregu. 00611.2010

Ullenhag, G. J., Mozaffari, F., Broberg, M., Mellstedt, H., and Liljefors, M. (2017). Clinical and immune effects of lenalidomide in combination with gemcitabine in patients with advanced pancreatic cancer. PLoS ONE 12:e169736. doi: 10.1371/journal.pone. 0169736
Vinogradova, Y., Coupland, C., and Hippisley-Cox, J. (2013). Exposure to bisphosphonates and risk of common non-gastrointestinal cancers: series of nested case-control studies using two primary-care databases. Br. J. Cancer 109, 795-806. doi: 10.1038/bjc.2013.383

Yadav, Y. C. (2019). Effect of cisplatin on pancreas and testies in wistar rats: biochemical parameters and histology. Heliyon 5:e02247. doi: 10.1016/j.heliyon.2019.e02247

Yasui-Furukori, N. (2012). Update on the development of lurasidone as a treatment for patients with acute schizophrenia. Drug Design Dev. Ther. 6, 107-115. doi: 10.2147/DDDT.S11180

Zhou, Y., Zhou, B., Pache, L., Chang, M., Khodabakhshi, A. H., Tanaseichuk, O., et al. (2019). Metascape provides a biologist-oriented resource for the analysis of systems-level datasets. Nat. Commun. 10:1523. doi: 10.1038/s41467-019-09234-6

Conflict of Interest: The authors declare that the research was conducted in the absence of any commercial or financial relationships that could be construed as a potential conflict of interest.

Copyright () 2020 Taguchi and Turki. This is an open-access article distributed under the terms of the Creative Commons Attribution License (CC BY). The use, distribution or reproduction in other forums is permitted, provided the original author(s) and the copyright owner(s) are credited and that the original publication in this journal is cited, in accordance with accepted academic practice. No use, distribution or reproduction is permitted which does not comply with these terms. 